\title{
A Review on Various Issues and Applications in Wireless Sensor Networks
}

\author{
Patil Yogita Dattatraya ${ }^{1}$, Jayashree Agarkhed ${ }^{2}$ \\ ${ }^{1} \mathrm{PhD}$ Scholar, Department of Computer Science \& Engineering, P.D.A College of Engineering, Kalaburagi, India \\ ${ }^{2}$ Professor, Department of Computer Science \& Engineering, P.D.A College of Engineering, Kalaburagi, India
}

\begin{abstract}
With the enormous advancement in the field of embedded computer and sensor technology, Wireless Sensor Networks (WSNs) have made remarkable impact in today's world. These WSNs consist of several thousands of sensor nodes deployed randomly, are capable of sensing, actuating, and communicating the collected information. Since wireless sensor networks are constrained by cost, scalability, topology change and power consumption, new technologies are being considered to overcome these and many other issues. This paper focuses on types of WSNs along with ways to overcome these challenges in respective WSNs and need of appropriate sensor for achieving specific application requirement. Use of efficient approach to achieve certain performance parameters has been highlighted.
\end{abstract}

Keywords: Wireless Sensor Network, Sensor types, Applications, Simulators, Performance metric.

\section{Introduction}

Wireless sensor networks (WSNs) have gained worldwide attention in recent years, particularly with the proliferation in Micro-Electro-Mechanical Systems (MEMS) technology which has facilitated the development of smart sensors. These sensors are small, with limited processing and computing resources, and they are inexpensive compared to traditional sensors. These sensor nodes can sense, measure, and gather information from the environment and, based on some local decision process, they can transmit the sensed data to the user. Smart sensor nodes are low power devices equipped with one or more sensors, a processor, memory, power supply, radio, and an actuator [1].

A variety of mechanical, thermal, biological, chemical, optical, and magnetic sensors may be attached to the sensor node to measure properties of the environment. Since the sensor nodes have limited memory and are typically deployed in difficult-to-access locations, a radio is implemented for wireless communication to transfer the data to a base station (e.g., a laptop, a personal handheld device, or an access point to a fixed infrastructure). Battery is the main power source in a sensor node. Secondary power supply that harvests power from the environment such as solar panels may be added.

A WSN typically has little or no infrastructure. It consists of a number of sensor nodes (few tens to thousands) working together to monitor a region to obtain data about the environment. Depending on the application and the type of sensors used, actuators may be incorporated in the sensors. Wireless sensor network overview presented in section 2 which include WSN architecture its applications and various issues. Literature review specified in section 3. Use of different simulators presented in section 4 along with different performance metric and conclusion given in section 5.

\section{Wireless Sensor Network}

Wireless Sensor Networks (WSNs) is the network comprised of a large number of nodes with sensing and routing capabilities. These capabilities are represented by considering WSN architecture discussed in next section.

\subsection{Architecture of WSN}

Wireless sensor network consist of huge number of sensor nodes that are spatially deployed in the areas of interest in order to monitor environmental and physical conditions. The basic structure of wireless sensor network is shown below in Figure 1.1.

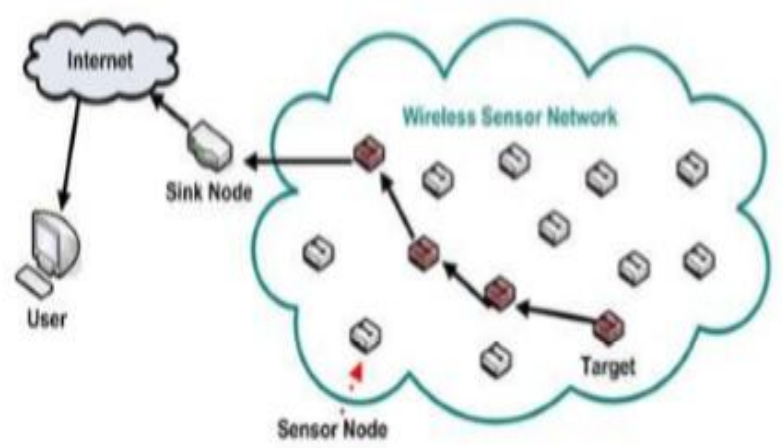

Figure 1.1: Basic architecture of Wireless Sensor Network

A wireless sensor network comprises of lightweight, small low power sensor nodes with the sensing, computational, and wireless communication capability. They are randomly or deterministic deployed in the area of interest. These sensor nodes communicate among themselves directly or to base station. Sensor field as shown above consist of large number of sensors nodes, each sensor nodes transmit its information to other neighbor node. Sink node collects the information and transmits to the user with the help of internet [2]. Depending on type of application requirement WSNs are further classified into various types as listed in next section. 


\section{International Journal of Science and Research (IJSR) \\ ISSN (Online): 2319-7064}

Index Copernicus Value (2013): 6.14 | Impact Factor (2014): 5.611

\subsection{Types of WSNs}

Current WSNs are deployed on land, underground, and underwater. Depending on application requirement Wireless
Sensor network is further classified into following types as shown in figure 1.2. below.

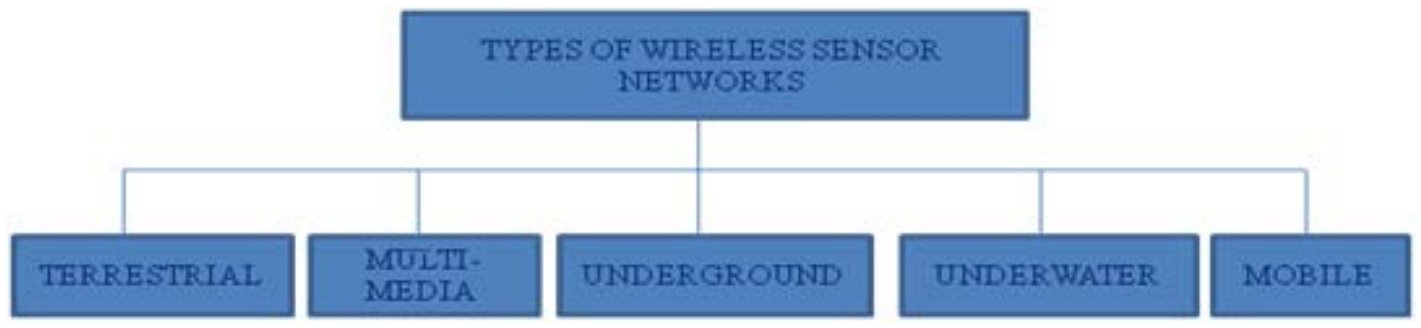

Figure 1.2: Types of wireless sensor networks

- Terrestrial WSNs: In ad hoc deployment, sensor nodes can be dropped from a plane and randomly placed into the target area. In pre-planned deployment, there is grid placement, optimal placement models. For a terrestrial WSN, energy can be conserved with multi-hop optimal routing, short transmission range, in-network data aggregation, eliminating data redundancy, minimizing delays, and using low duty-cycle operations [3].

- Underground WSNs: It consist of a number of sensor nodes buried underground or in a cave or mine used to monitor underground conditions. Underwater sensor nodes must be able to self-configure and adapt to harsh ocean environment $\&$ are equipped with a limited battery which cannot be replaced. The issue of energy conservation for underwater WSNs involves developing efficient underwater communication and networking techniques [3].

- Mobile WSNs: It consists of a collection of sensor nodes that can move on their own and interact with the physical environment. Mobile nodes have the ability to sense, compute, communicate like static nodes and have ability to reposition and organize itself in the network. Challenges in mobile WSN include deployment, localization, self-organization, navigation and control, coverage, energy, maintenance, data process and dynamic routing [3].

- Underwater WSNs: In these, sensor nodes and vehicles are located underwater. Autonomous vehicles are used for gathering the data from the sensor nodes. Sparse deployment of nodes is done in this network. Main problems that come under this while communicating are limited bandwidth, long propagation delay and signal fading issue [3].

- Multi-media WSNs: It monitors and tracks events in the form of multimedia such as video, audio, and imaging. Multi-media WSNs consist of a number of low cost sensor nodes equipped with cameras and microphones. Challenges in multi-media WSN include high bandwidth demand, high energy consumption, quality of service (Qos) provisioning, data processing and compressing techniques, and cross-layer design. Similarly, cross-layer interaction among the layers can improve the processing and the delivery process [4].

Data for smart environments are obtained through Wireless Sensor Networks, where thousands of sensors are deployed at different locations operating in different modes [5]. A sensor is capable of sensing different type of data and used according to application requirement. This leads to classification of sensors according to sensed data as discussed in next section.

\subsection{Types of Sensors}

There are three main categories of sensors as Physical, chemical and biological sensors. The table 1.1 below specifies different types of sensors, their application and measuring parameter. 
International Journal of Science and Research (IJSR)

ISSN (Online): 2319-7064

Index Copernicus Value (2013): 6.14 | Impact Factor (2014): 5.611

Table 1.1: Sensor type, its measuring parameter and applications

\begin{tabular}{|c|c|c|}
\hline $\begin{array}{l}\text { Sensor } \\
\text { type }\end{array}$ & Measuring parameter & Applications \\
\hline $\begin{array}{l}\text { Environm } \\
\text { ental }\end{array}$ & $\begin{array}{l}\text { Temperature, } \\
\text { (soil,leaf,ambient), Soil moisture, } \\
\text { Wind (speed and direction), } \\
\text { Pressure,Leaf, } \mathrm{Ph} \text {, Redox }\end{array}$ & $\begin{array}{l}\text { - Precision agricultural applications required in the terms of measuring temperature, } \\
\text { humidity and wind. } \\
\text { - Ph and Redox sensors being demanded for water quality }\end{array}$ \\
\hline Gas & $\begin{array}{c}\mathrm{Co} 2, \mathrm{Co}, \mathrm{CH} 4, \mathrm{O} 2, \mathrm{NH} 3, \mathrm{SH} 2, \\
\text { NO2, Pollution }\end{array}$ & $\begin{array}{l}\text { - Organic gases (carbon) derived from the "live systems" such as } \\
\text { respiration in humans (CO2), animal (CH4) and Combustion (CO) } \\
\text { of vegetable elements (fire forest) are the most required sensors } \\
\text { - Other toxic gases which can be found in animal farms (NH3, } \\
\text { SH2) and the fabric and cars pollution gases (NO2) complete the list. }\end{array}$ \\
\hline Physical & $\begin{array}{l}\text { Accelerometer, Presence, } \\
\text { Vibration, Power, Hall, } \\
\text { Ultrasound, Water, Sound, Bend, } \\
\text { Flex, Strain, Stress }\end{array}$ & $\begin{array}{l}\text { - Motion of any kind using accelerometers, vibration, and presence sensors . } \\
\text { - Security applications } \\
\text { - Bend, flex, strain and stress sensors let know how each object is } \\
\text { interacting with the world and monitories its state. }\end{array}$ \\
\hline Optical & $\begin{array}{l}\text { Infrared, Sunlight, Radiation, } \\
\text { Ultraviolet, color }\end{array}$ & $\begin{array}{l}\text { - Optical sensors to detect } \\
\text { human presence through } \\
\text { the IR spectrum. } \\
\text { - Agriculture applications } \\
\text { where the sun light, radiation and ultraviolet, sensors are required in order to } \\
\text { measure the total amount of energy and light which is absorbed by the plants. }\end{array}$ \\
\hline Biometric & $\begin{array}{l}\text { Electrocardiogram ECG, } \\
\text { Oximetry, Pulse, Fall, Sweat }\end{array}$ & $\begin{array}{l}\text { - Prevent a possible attack or the fall of a elderly person (using an accelerometer) } \\
\text { by monitoring his heart pulse, rate and other heart activities. Used in combination of } \\
\text { SMS alarms using the GSM/GPRS module }\end{array}$ \\
\hline
\end{tabular}

Thus sensors can be used in wide range of applications related to different category as given in next section.

\subsection{Applications}

WSN applications can be classified into two categories monitoring and tracking. Monitoring applications include indoor/outdoor environmental monitoring, health and wellness monitoring, power monitoring, inventory location monitoring, factory and process automation, and seismic and structural monitoring. Tracking applications include tracking objects, animals, humans, and vehicles. Many different applications that have been deployed and tested in the real environment are discussed as below:

- PinPtr system is experimental counter-sniper system developed to detect and locate shooters. Sensors detect and measure the time of arrival of muzzle blasts and shock waves from a shot and route their measurements to a base station to compute the shooter's location [6].

- Macroscope of redwood monitors and records the redwood trees in Sonoma, California. Each sensor node measures air temperature, relative humidity, and photosynthetically-active solar radiation. Sensor nodes are placed at different heights of the tree [7].

- Underwater monitoring used to monitoring of coral reefs and fisheries. The sensor network consists of static and mobile underwater sensor nodes. The nodes communicate via point-to-point links using high speed optical communications. Nodes broadcast using acoustic protocol integrated in the TinyOS protocol stack [8].

- Volcanic monitoring with WSN can help accelerate the deployment, installation, and maintenance process. The challenges of a WSN application for volcanic data collection include reliable event detection, efficient data collection, high data rates, and sparse deployment of nodes [9].

The listed application try to achieve one or more performance parameters as discussed in next section.

\subsection{Issues in WSN}

The various issues that must be addressed in WSN are providing high bandwidth, low energy consumption, QoS provisioning, node mobility, congestion control, congestion detection, congestion mitigation, reliability measure, end-toend / hop-by-hop packet recovery, cache ACK/NACK, scalability, synchronization, data cache, data aggregation, computational overhead, data security, availability, authorization, authentication, confidentiality, integrity, non repudiation, freshness $[10,11,12]$. The existing systems that are developed for one of the application specified in section 2.3 or way to overcome one or more above issues are mentioned in literature survey papers as mentioned in next section.

\section{Literature Survey}

Existing system and their limitation are analyzed by following literature survey. In [1] author Jennifer Yicket proposed that the design of a WSN depends significantly on the application, and it must consider fact ors such as the environment, the application's design objectives, cost, hardware, and system constraints. Here problem is classified into three different categories (i) internal platform and underlying operating system, (ii) communication protocol stack, and (iii) network services, provisioning, and deployment. The review of major development in these three categories and outline of new challenges has been given. In [2] author presents energy conservation schemes and techniques for data acquisition.

Aashima Singla et al [3] specified need of security in sensor networks, security issues and various DoS attacks on different layers. Security is an important requirement and complicates enough to set up in different domains of WSN. Here author discuss various dimensions of security (availability, integrity, confidentiality and authenticity) that are being directed by different physical attacks. Monika Jena 


\section{International Journal of Science and Research (IJSR) \\ ISSN (Online): 2319-7064 \\ Index Copernicus Value (2013): 6.14 | Impact Factor (2014): 5.611}

et al [4] proposed for fulfilling real- time requirements for multimedia delivery of data, new protocols need to be developed which not only ensure bounded response time but also strive to minimize energy consumption in data processing and communication. In this various QoS issues in network layer in WMSN is specified and explains the reasons for the failure of traditional QoS models in WMSN.

In [5] author presented a review on the various research issues involved in the WSN applications. Further author G. Simon et al in [6] proposed counter-sniper system developed to detect and locate shooters. Here middleware services developed on TinyOS include time synchronization, message routing with data aggregation, and localization. G. Tolle et al [7] proposed that Macroscope of redwood is used to monitor and record the redwood trees in Sonoma, California. Each sensor node measures air temperature, relative humidity, and photo-synthetically-active solar radiation. Vasilescu et al [8] presents a sensor network that contain static and mobile underwater sensor nodes. These nodes communicate via point-to-point links using high speed optical communications. Mobile nodes can locate and move above the static nodes to collect data and perform work maintenance functions for deployment, re-location, and recovery.

G. Werner-Allen et al [9] proposed that when an interesting event occurs, the node will route a message to the base station. If multiple nodes report the same event, then data is collected from the nodes in a round-robin fashion. When data collection is completed, the nodes return to sampling and storing sensor data locally. Further to improve the node lifetime and to reduce end-to-end delay proper routing protocol must be used. Kemal Akkaya et al [10] has proposed recent routing protocols for sensor networks and presents a classification for the various approaches pursued. The three main categories explored are data-centric, hierarchical and location-based. Each routing protocol is described and discussed under the appropriate category. Moreover, protocols using contemporary methodologies such as network flow and quality of service modeling are also discussed.

Preeti Sharma [11] proposed that sensor network provides endless opportunities but at same time pose various challenges as there are resource constraints like energy i.e. it has limited battery life, memory computation etc. The sensor network deploy in hostile environment which increase the probability of being attack by adversary. The wireless communication technology also acquire various types of security threats, it is very important to understand the security challenges and issues in order to design appropriate security mechanisms. Also Gaurav Sharma et al [12] proposed that WSN suffer from many constraints including lower processing power, low battery life, small memory and wireless communication channel, security becomes the main concern to deal with such kind of networks. Due to these well accepted limitations, WSN is not able to deal with traditional cryptographic algorithms. The author gives overview of cryptographic frameworks designed so far and also a comparison of existing schemes is tabled

Fanf-Jing et al [13] proposed that cyber physical system (CPS) has emerged as a promising direction to enrich the interactions between physical and virtual worlds. Cyberphysical systems bridge the cyber world (e.g., information, communication, and intelligence) to the physical world through lots of sensors and actuators. A CPS may consist of multiple static/mobile sensor and actuator networks integrated under an intelligent decision system. CPS platforms and systems include health care, navigation, rescue, intelligent transportation, social networking, and gaming applications. In author specifies how CPS applications exploit the physical information collected by WSNs to bridge real and cyber space.

Salem Hadim et al [14] proposed that middleware used to bridge the gap between applications and low-level constructs is a novel approach to resolve many wireless sensor network issues and to enhance application development. The survey discusses representative WSN middleware, presenting the state of the research.

Nathalie Mitton et al [15] proposed that constantly growing number of ever more powerful devices (smartphones, sensors, household appliances, RFID devices, etc.) join the Internet, significantly impacting the global traffic volume (data sharing, voice, multimedia, etc.) and foreshadowing a world of (more or less) smart devices, or "things" in the Internet of Things (IoT) perspective. Here author specify how sensors and actuators can be discovered and aggregated by applying the Cloud provisioning model.

From the review of papers, we analyzed that different approach can be used to achieve different performance metric using different types of simulators as specified in next section.

\section{Simulators and Performance Metric}

The performance of system can be improved by efficient design of routing protocols, scheduling methods using different approaches like clustering method, Cross-layer approach, Middleware support, integration into Internet of things or Sensor-Cloud integration. Performance of system can evaluated using simulation tools such as NS2,OMNET, OPNET, MATLAB, QUALNET, CLOUDSIM etc. considering various metrics as listed in Table 1.2. 


\section{International Journal of Science and Research (IJSR) \\ ISSN (Online): 2319-7064}

Index Copernicus Value (2013): 6.14 | Impact Factor (2014): 5.611

Table 1.2: List of performance metric

\begin{tabular}{|c|c|c|}
\hline Performance metric & Expressed in & Description \\
\hline BER (Bit error rate) & $\%$ & Number of bits error divided by total number of transmitted bits in a specified time interval \\
\hline $\begin{array}{l}\text { Average end-to-end } \\
\text { delay }\end{array}$ & Seconds & It is time taken by a data packet to be transmitted across network from source to destination \\
\hline Packet Delivery ratio & $\begin{array}{l}\text { Number of } \\
\text { packets }\end{array}$ & $\begin{array}{l}\text { It is ratio of total number of delivered packets successfully received by sink node to the no. } \\
\text { of packets sent by all sensor nodes in network }\end{array}$ \\
\hline Energy c & KJ & $\begin{array}{l}\text { It is measure at which energy is dissipated by sensor nodes in network within a specified } \\
\text { time period }\end{array}$ \\
\hline Network 1 & Minutes & $\begin{array}{l}\text { Lifetime of WSN defined as the time elapsed until the first node dies, the last node dies or a } \\
\text { fraction of nodes dies }\end{array}$ \\
\hline $\begin{array}{l}\text { Average network } \\
\text { Throughput }\end{array}$ & Bits per second & It is average number of data packets successfully received by the sink node per unit time \\
\hline
\end{tabular}

The above parameters can be evaluated to satisfy specific application requirements in real time environment using above mentioned simulators.

\section{Conclusion}

Wireless Sensor Network is ever green technology forever due its wide range of applications in various fields. For achieving excellent application, one has to consider various challenges in WSNs. In this paper we highlighted challenges, use of appropriate sensor for measuring unambiguous parameter in the environment along with different performance metric to be considered.

\section{References}

[1] Jennifer Yick, Biswanath Mukherjee, Dipak Ghosal, “ Wireless sensor network survey," Computer Networks 52, pp. 2292-2330, 2008.

[2] Giuseppe Anastasi, Marco Conti, Mario Di Francesco, Andrea Passarella, "Energy Conservation in wireless sensor networks : A survey," Ad Hoc Networks7(2009) 537-568, Elsevier 2008.

[3] Aashima Singla, Ratika Sachdeva, "Review on Security Issues and Attacks in Wireless Sensor Networks," International Journal of Advanced Research in Computer Science and Software Engineering, Vol. 3, April 2013.

[4] Monika Jena ," QoS Provisioning issues in Wireless Multimedia Sensor Networks," 2009.

[5] Edwin Prem Kumar Gilbert, Baskaran Kaliaperumal, and Elijah Blessing Rajsingh," Research Issues in Wireless Sensor Network Applications: A Survey," International Journal of Information and Electronics Engineering, Vol. 2, No. 5, September 2012.

[6] G. Simon, M. Maroti, A. Ledeczi, G. Balogh, B. Kusy, A. Nadas, G. Pap, J. Sallai, K. Frampton, "Sensor network-based counter sniper system", Proc. Second International Conference on Embedded Networked Sensor Systems, Baltimore, MD, 2004.

[7] G. Tolle, J. Polastre, R. Szewczyk, D. Culler, N. Turner, K. Tu, S. Burgess, T. Dawson, P. Buonadonna, D. Gay, W. Hong, "A macroscope in the redwoods," in Proc. Third International Conference on Embedded Networked Sensor Systems, San Diego, CA, 2005.

[8] I. Vasilescu, K. Kotay, D. Rus, M. Dunbabin, P. Corke, "Data collection, storage, retrieval with an underwater sensor network," Proc. of the Third
International Conference on Embedded Networked Sensor Systems, San Diego, CA, 2005.

[9] G. Werner-Allen, K. Lorincz, M. Welsh, O. Marcillo, J. Johnson, M.Ruiz, J. Lees, "Deploying a wireless sensor network on an active volcano," IEEE Internet Computing 10, pp.18-25, 2006.

[10]Kemal Akkaya, Mohamed Younis, "A survey on routing protocols for wireless sensor networks," Adhoc Networks 3, pp. 325-349, 2005.

[11] Preeti Sharma, "A Review of Attacks on Wireless Sensor Networks," Information Systems and Communication, Vol. 3, pp. 251-255, 2012.

[12] Gaurav Sharma, Suman Bala, Anil K. Verma, "Security Frameworks for Wireless Sensor NetworksReview," in Proc. $2^{\text {nd }}$ International Conference on Communication, Computing \&amp., vol 6, 2012.

[13]Fang-Jing Wua, Yu-Fen Kaob, Yu-Chee Tseng a, Zenon Chaczko, Venkatesh Mahadevan," From wireless sensor networks towards cyber physical systems," Pervasive and Mobile Computing, 2011.

[14] Salem Hadim, Nader Mohamed, "Middleware: Middleware Challenges and Approaches for Wireless Sensor Networks," IEEE Computer Society Vol. 7, No. 3; March 2006.

[15] Nathalie Mitton, Symeon Papavassiliou, Antonio Puliafito and Kishor S Trivedi, "Combining Cloud and sensors in a smart city environment," EURASIP Journal on Wireless Communications and Networking, pp 247-257, 2012. 